\title{
Odd Harmonious Labeling on Pleated of the Dutch Windmill Graphs
}

\author{
Fery Firmansah', Muhammad Ridlo Yuwono² \\ 1, 2Mathematics Edu. Depart. University of Widya Dharma Klaten, Indonesia
}

Email: feryfirmansah@unwidha.ac.id, ridloyuwono@unwidha.ac.id

\begin{abstract}
A graph $G(V(G), E(G))$ is called graph $G(p, q)$ if it has $p=|V(G)|$ vertices and $q=|E(G)|$ edges. The graph $G(p, q)$ is said to be odd harmonious if there exist an injection $f: V(G) \rightarrow\{0,1,2, \ldots, 2 q-1\}$ such that the induced function $f^{*}: E(G) \rightarrow\{1,3,5, \ldots, 2 q-1\}$ defined by $f^{*}(u v)=f(u)+f(v)$. The function $f^{*}$ is a bijection and $f$ is said to be odd harmonious labeling of $G(p, q)$. In this paper we prove that pleated of the Dutch windmill graphs $C_{4}^{(k)}(r)$ with $k \geq 1$ and $r \geq 1$ are odd harmonious graph. Moreover, we also give odd harmonious labeling construction for the union pleated of the Dutch windmill graph $C_{4}^{(k)}(r) \cup C_{4}^{(k)}(r)$ with $k \geq 1$ and $r \geq 1$.
\end{abstract}

Keywords: odd harmonious labeling, pleated graph, the Dutch windmill graph

\section{INTRODUCTION}

In this paper we consider simple, finite, connected and undirected graph. A graph $G(p, q)$ with $p=|V(G)|$ vertices and $q=|E(G)|$ edges. A graph labeling which has often been motivated by practical problems is one of fascinating areas of research. Labeled graphs serves as useful mathematical models for many applications in coding theory, communication networks, and mobile telecommunication system. We refer to Gallian [1] for a dynamic survey of various graph labeling problems along with extensive bibliography. Most graph labeling methods trace their origin to one introduced by Rosa in 1967, or one given by Graham and Sloane in 1980.

Graham and Sloane [2] introduced and defined harmonious labeling as follows:

Definition 1. A graph $G(p, q)$ is said to be harmonious if there is an exist injection $f: V(G) \rightarrow Z_{q}$ such that the induced function $f^{*}: E(G) \rightarrow Z_{q}$ defined by $f^{*}(u v)=(f(u)+f(v))(\bmod q)$ is a bijection and $f$ is said to be harmonious labeling of $G(p, q)$.

Liang and Bai [3] introduced and defined odd harmonious labeling as follows:

Definition 2. A graph $G(p, q)$ is said to be odd harmonious if there is an exist injection $f: V(G) \rightarrow$ $\{0,1,2, \ldots, 2 q-1\}$ such that the induced function $f^{*}: E(G) \rightarrow\{1,3,5, \ldots, 2 q-1\}$ defined by $f^{*}(u v)=$ $f(u)+f(v)$ is a bijection and $f$ is said to be odd harmonious labeling of $G(p, q)$.

A graph that admits odd harmonious labeling is called odd harmonious graphs. Liang and Bai [3] have obtained the necessary conditions for the existence of odd harmonious labeling graph. They proved if $G$ is an odd harmonious graph, then $G$ is a bipartite graph and $G(p, q)$ is an 
odd harmonious labeling then number vertices is bounded by $\sqrt{q} \leq p \leq 2 q-1$. The maximal label of all vertices in an odd harmonious graph $G$ is at most $2 q-\delta(G)$, where $\delta(G)$ is the minimum degree of the vertices of $G$. In the same paper Liang and Bai [3] proved that $C_{n}$ is odd harmonious if and only if $n \equiv 0(\bmod 4)$ and a complete graph $K_{n}$ is odd harmonious if and only if $n=2$. The odd harmoniousness of graph is useful for the solution of undetermined equations.

Alyani, Firmansah, Giyarti and Sugeng [4] proved the odd harmonious labeling of $k C_{n}$ snake graphs for specific values of $n$, that is, for $n=4$ and $n=8$. Firmansah [5] proved that union of snake graph $k C_{4} \cup k C_{4}$ with $k \geq 1$ and pleated of snake graph $k C_{4}(r)$ with $k \geq 1$ and $r \geq 1$ admitted odd harmonious graph. Firmansah and Sugeng [6] proved that the Dutch wind mill graph $C_{4}^{(k)}$ with $k \geq 1$ and union of the ducth windmill graph $C_{4}^{(k)} \cup C_{4}^{(k)}$ with $k \geq 1$ admited odd harmonious labeling. Firmansah [7] proved that the quadrilateral windmill graph $D Q^{(k)}$ with $k \geq$ 1 admitted odd harmonious graph. Several results have been published on odd harmonious labeling see [8], [9], [1], [10], [11], [12], [13], and [14].

In this paper, we prove that pleated of the Dutch windmill graphs $C_{4}^{(k)}(r)$ with $k \geq 1$ and $r \geq 1$ are the odd harmonious graph. Moreover, we also give the odd harmonious labeling construction for the union pleated of the Dutch windmill graph $C_{4}^{(k)}(r) \cup C_{4}^{(k)}(r)$ with $k \geq 1$ and $r \geq 1$.

Definition 3. [6] The Dutch windmill graphs $C_{4}^{(k)}$ with $k \geq 1$ is a graph which consists of $k$ copies of cycle graphs $C_{4}$ with a common central vertex $u_{0}$.

Definition 4. [5] The pleated cycle graphs $C_{4}(r)$ with $r \geq 1$ is a graph formed from cycle graphs $C_{4}$ with vertex set $\left\{u_{0}, v_{1}, v_{2}, u_{1}\right\}$ by adding $\left\{u_{2}, u_{3}, \ldots, u_{r}\right\}$ vertices which are connected with vertex $v_{1}$ and $v_{2}$.

\section{RESULTS AND DISCUSSIONS}

We have some observation to show the result of odd harmonious graph.

Definition 5. The pleated of the Dutch windmill graphs $C_{4}^{(k)}(r)$ with $k \geq 1$ and $r \geq 1$ is a graph which consists of $k$ copies of the pleated cycle graphs $C_{4}(r)$ with a common central vertex $u_{0}$.

The vertex notation and construction of the pleated cycle graph $C_{4}(r)$ with $r \geq 1$ and pleated of the Dutch windmill graph $C_{4}^{(k)}(r)$ with $k \geq 1$ and $r \geq 1$ is shown in Figure 1 .

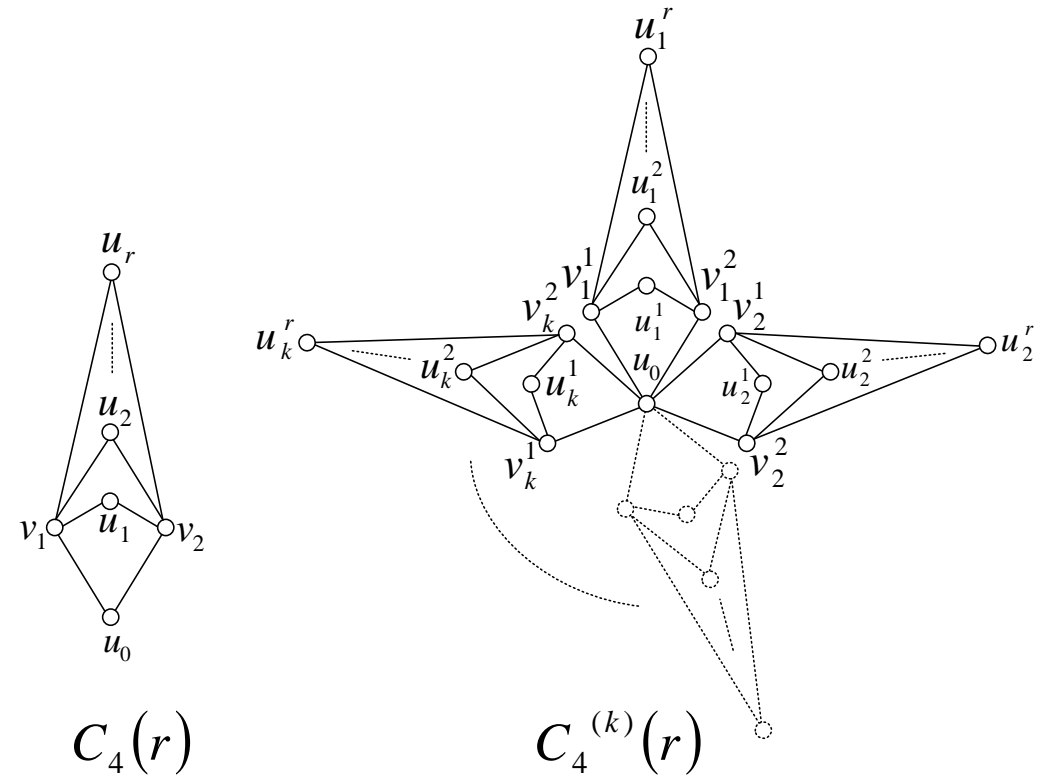

Figure 1. The pleated cycle graph $C_{4}(r)$ and pleated of the Dutch windmill graph $C_{4}^{(k)}(r)$ 
Definition 6. A graphs $C_{4}^{(k)}(r) \cup C_{4}^{(k)}(r)$ with $k \geq 1$ and $r \geq 1$ is a union pleated of the Dutch windmill graphs $C_{4}^{(k)}(r)$ with $k \geq 1$ and $r \geq 1$.

The vertex notation and construction the union pleated of the Dutch windmill graphs $C_{4}^{(k)}(r) \cup C_{4}^{(k)}(r)$ with $k \geq 1$ and $r \geq 1$ is shown in Figure 2 .

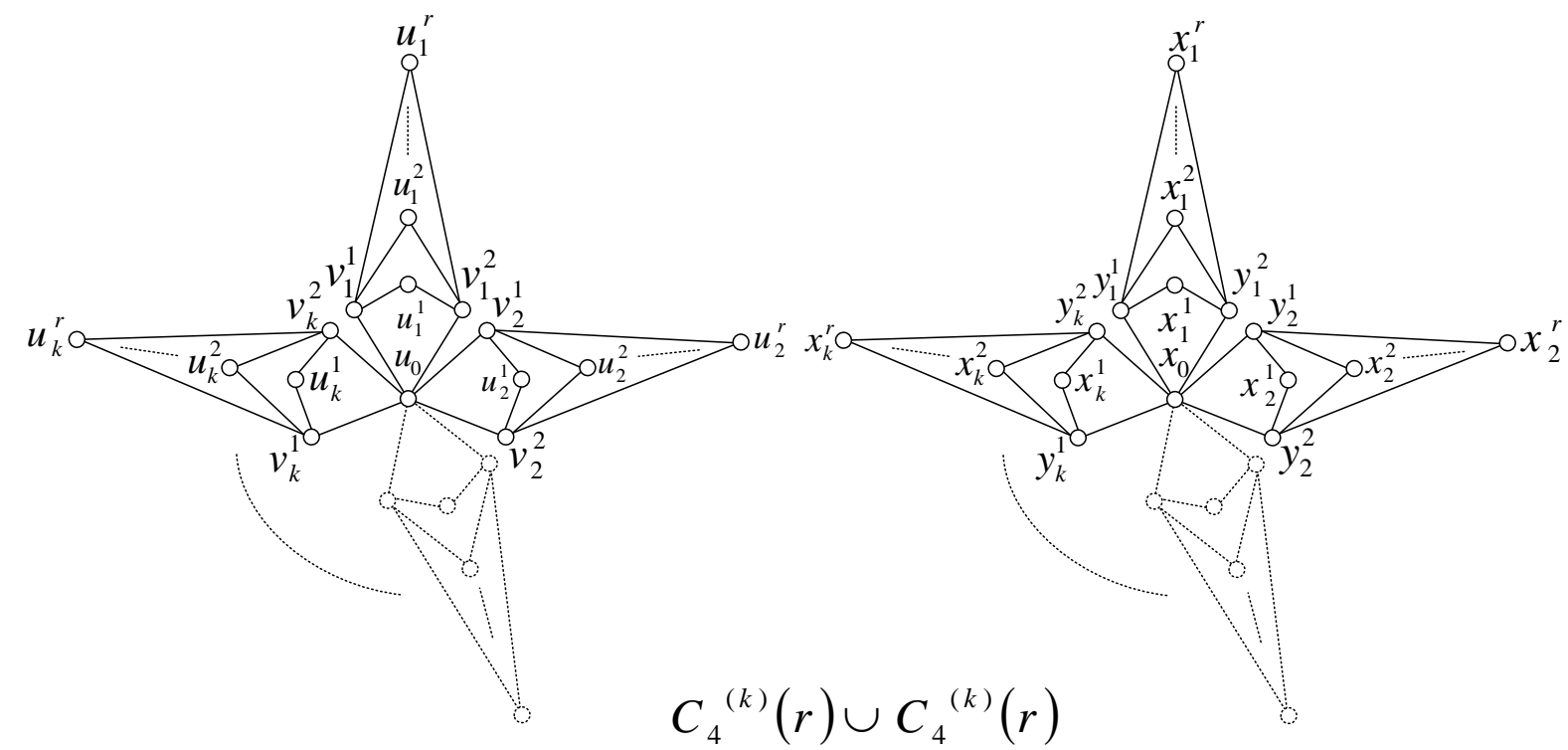

Figure 2. The union pleated of the Dutch windmill graphs $C_{4}^{(k)}(r) \cup C_{4}^{(k)}(r)$

Theorem 1. The pleated of the Dutch windmill graphs $C_{4}^{(k)}(r)$ with $k \geq 1$ and $r \geq 1$ is an odd harmonious graph.

Proof.

Let $\mathrm{G}$ be the pleated of the Dutch windmill graphs $C_{4}^{(k)}(r)$ with $k \geq 1$ and $r \geq 1$.

The vertex set and edge set of $C_{4}^{(k)}(r)$ are defined as follows

$V(G)=\left\{u_{0}\right\} \cup\left\{v_{i}{ }^{j} \mid 1 \leq i \leq k, j=1,2\right\} \cup\left\{u_{i}{ }^{m} \mid 1 \leq i \leq k, 1 \leq m \leq r\right\}$ and

$E(G)=\left\{u_{0} v_{i}{ }^{j} \mid 1 \leq i \leq k, j=1,2\right\} \cup\left\{v_{i}{ }^{j} u_{i}{ }^{m} \mid 1 \leq i \leq k, 1 \leq m \leq r, j=1,2\right\}$ then

$p=|V(G)|=k r+2 k+1$ and $q=|E(G)|=2 k r+2 k$.

Define the vertices labels $f: V(G) \rightarrow\{0,1,2,3 \ldots, 4 k r+4 k-1\}$ as follows

$$
\begin{aligned}
& f\left(u_{0}\right)=0 \\
& f\left(v_{i}^{j}\right)=4 i+2 j-5,1 \leq i \leq k, j=1,2 \\
& f\left(u_{i}^{m}\right)=(4 r+4) k-(4 r+4) i+4 m, 1 \leq i \leq k, 1 \leq m \leq r
\end{aligned}
$$

The labeling $f$ will induce the mapping $f^{*}: E(G) \rightarrow\{1,3,5,7, \ldots, 4 k r+4 k-1\}$ which is defined by $f^{*}(u v)=f(u)+f(v)$. Thus we have the edges labels as follows

$$
\begin{aligned}
& f^{*}\left(u_{0} v_{i}{ }^{j}\right)=4 i+2 j-5,1 \leq i \leq k, j=1,2 \\
& f^{*}\left(v_{i}^{j} u_{i}^{m}\right)=(4 r+4) k-4 r i+2 j+4 m-5,1 \leq i \leq k, 1 \leq m \leq r, j=1,2
\end{aligned}
$$

It is not difficult to show that the mapping $f$ is an injective mapping and the mapping $f^{*}$ admits a bijective mapping. Hence pleated of the Dutch windmill graphs $C_{4}^{(k)}(r)$ with $k \geq 1$ and $r \geq 1$ is an odd harmonious graph. 
Theorem 2. The union pleated of the Dutch windmill graphs $C_{4}^{(k)}(r) \cup C_{4}^{(k)}(r)$ with $k \geq 1$ and $r \geq$ 1 is an odd harmonious graph.

Proof.

Let $G$ be the union pleated of the Dutch windmill graphs $C_{4}^{(k)}(r) \cup C_{4}^{(k)}(r)$ with $k \geq 1$ and $r \geq 1$. The vertex set and edge set of $G$ are defined as follows

$V(G)=\left\{u_{0}\right\} \cup\left\{v_{i}{ }^{j} \mid 1 \leq i \leq k, j=1,2\right\} \cup\left\{u_{i}{ }^{m} \mid 1 \leq i \leq k, 1 \leq m \leq r\right\} \cup\left\{x_{0}\right\} \cup$

$\left\{y_{i}{ }^{j} \mid 1 \leq i \leq k, j=1,2\right\} \cup\left\{x_{i}{ }^{m} \mid 1 \leq i \leq k, 1 \leq m \leq r\right\}$ and

$E(G)=\left\{u_{0} v_{i}{ }^{j} \mid 1 \leq i \leq k, j=1,2\right\} \cup\left\{v_{i}^{j} u_{i}^{m} \mid 1 \leq i \leq k, 1 \leq m \leq r, j=1,2\right\} \cup$

$\left\{x_{0} y_{i}{ }^{j} \mid 1 \leq i \leq k, j=1,2\right\} \cup\left\{y_{i}{ }^{j} x_{i}{ }^{m} \mid 1 \leq i \leq k, 1 \leq m \leq r, j=1,2\right\}$

then $p=|V(G)|=2 k r+4 k+2$ and $q=|E(G)|=4 k r+4 k$.

Define the vertices labels $f: V(G) \rightarrow\{0,1,2,3 \ldots, 8 k r+8 k-1\}$ as follows

$$
\begin{aligned}
& f\left(u_{0}\right)=0 \\
& f\left(v_{i}^{j}\right)=4 i+2 j-5,1 \leq i \leq k, j=1,2 \\
& f\left(u_{i}^{m}\right)=(4 r+4) k-(4 r+4) i+4 m, 1 \leq i \leq k, 1 \leq m \leq r \\
& f\left(x_{0}\right)=2 \\
& f\left(y_{i}^{j}\right)=(4 r+4) k+4 i+2 j-7,1 \leq i \leq k, j=1,2 \\
& f\left(x_{i}^{m}\right)=(4 r+4) k-(4 r+4) i+6 m, 1 \leq i \leq k, 1 \leq m \leq r
\end{aligned}
$$

The labeling $f$ will induce the mapping $f^{*}: E(G) \rightarrow\{1,3,5,7, \ldots, 8 k r+8 k-1\}$ which is defined by $f^{*}(u v)=f(u)+f(v)$. Thus we have the edges labels as follows

$$
\begin{aligned}
& f^{*}\left(u_{0} v_{i}^{j}\right)=4 i+2 j-5,1 \leq i \leq k, j=1,2 \\
& f^{*}\left(v_{i}^{j} u_{i}^{m}\right)=(4 r+4) k-4 r i+2 j+4 m-5,1 \leq i \leq k, 1 \leq m \leq r, j=1,2 \\
& f^{*}\left(x_{0} y_{i}^{j}\right)=(4 r+4) k+4 i+2 j-5,1 \leq i \leq k, j=1,2 \\
& f^{*}\left(y_{i}^{j} x_{i}^{m}\right)=(8 r+8) k-4 r i+2 j+6 m-7,1 \leq i \leq k, 1 \leq m \leq r, j=1,2
\end{aligned}
$$

It is not difficult to show that the mapping $f$ is an injective mapping and the mapping $f^{*}$ admits a bijective mapping. Hence union pleated of the Dutch windmill graphs $C_{4}^{(k)}(r) \cup C_{4}^{(k)}(r)$ with $k \geq$ 1 and $r \geq 1$ is an odd harmonious graph.

Example 1. Odd harmonious labeling for pleated of the Dutch windmill graph $C_{4}^{(5)}(4)$ is shown in Figure 3.

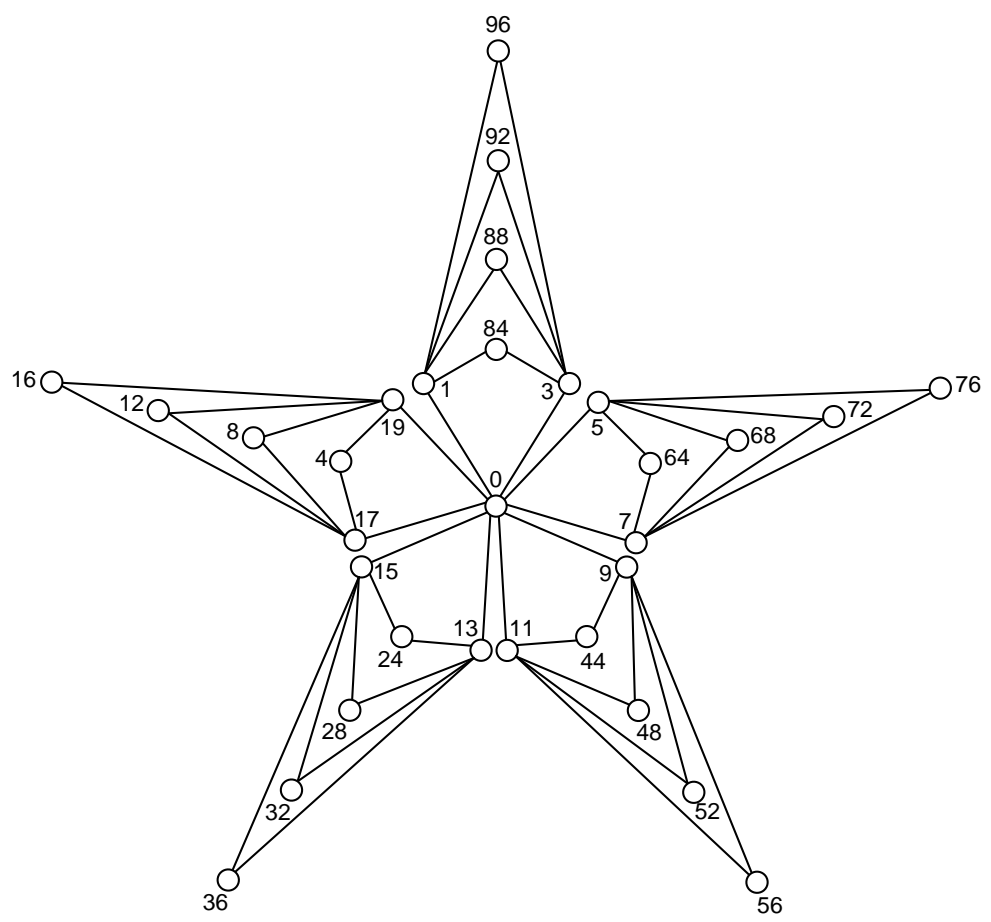

Figure 3. Pleated of the Dutch windmill graph $C_{4}^{(5)}(4)$ and its odd harmonious labeling. 
Example 2. Odd harmonious labeling for the union pleated of the Dutch windmill graphs $C_{4}^{(4)}(3) \cup C_{4}^{(4)}(3)$ and $C_{4}^{(5)}(4) \cup C_{4}^{(5)}(4)$ is shown in Figure 4.
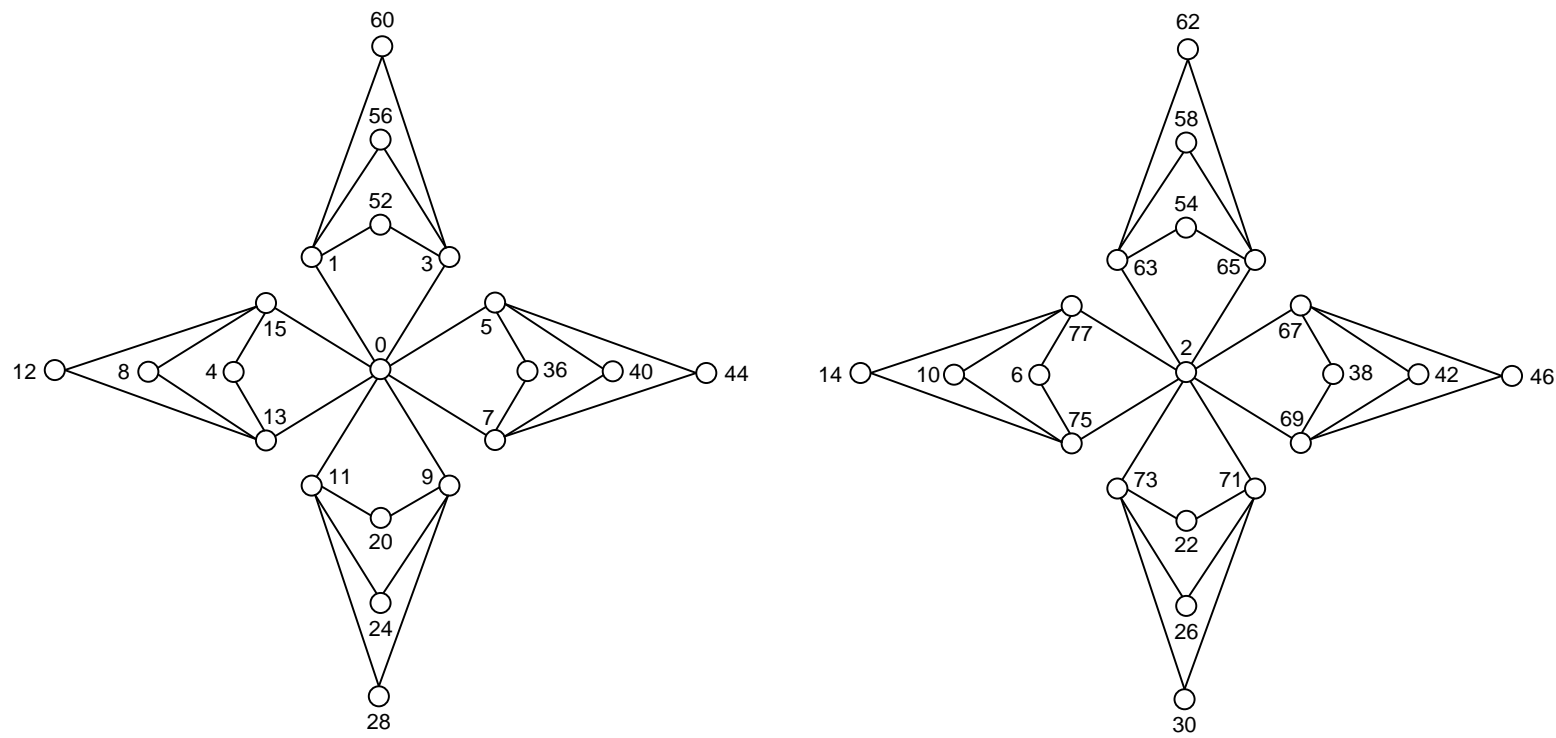

$C_{4}^{(4)}(3) \cup C_{4}^{(4)}(3)$
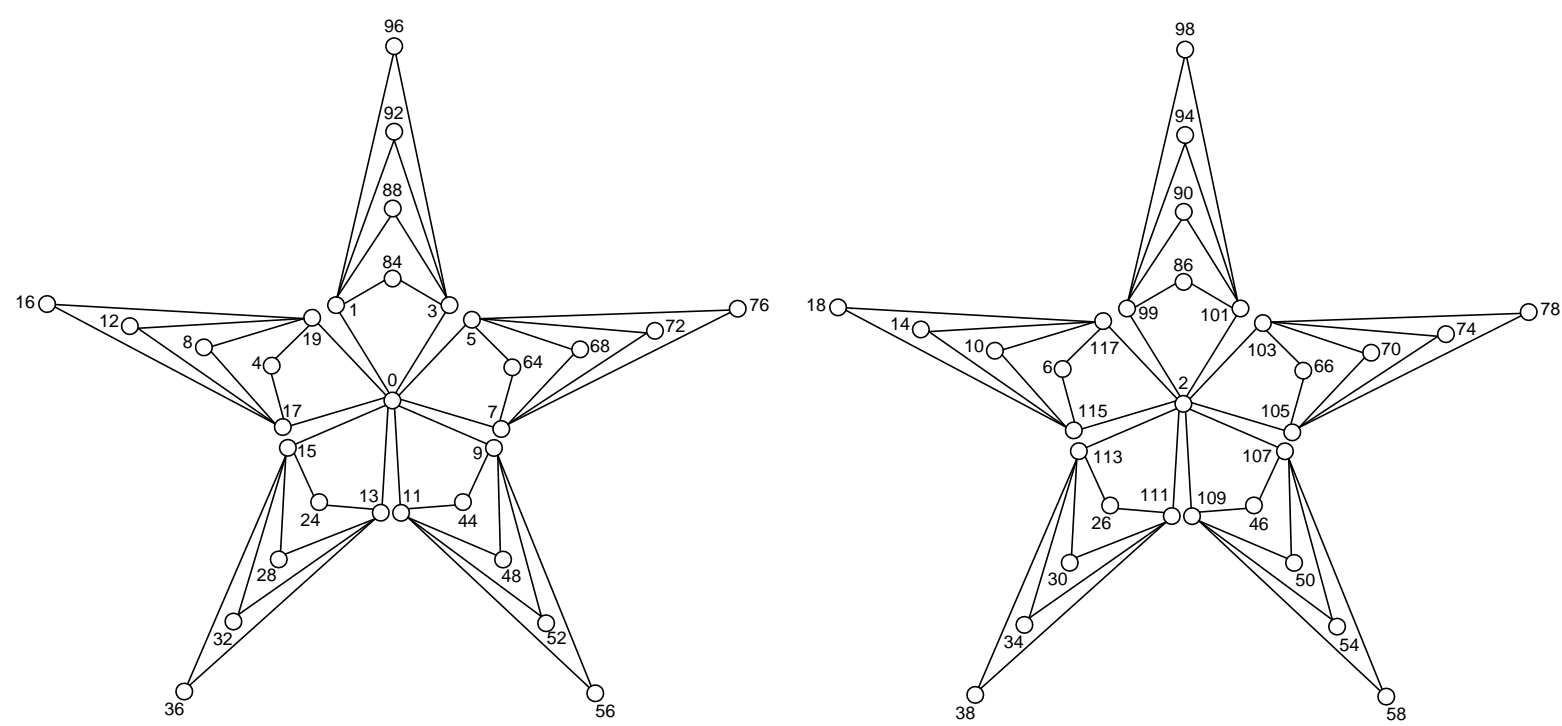

$C_{4}^{(5)}(4) \cup C_{4}^{(5)}(4)$

Figure 4. The union pleated of the Dutch windmill graph $C_{4}^{(4)}(3) \cup C_{4}^{(4)}(3)$,

$C_{4}^{(5)}(4) \cup C_{4}^{(5)}(4)$ and its odd harmonious labeling.

\section{CONCLUSION}

We have shown that the pleated of the Dutch windmill graphs and the union pleated of the Dutch windmill graphs admitted odd harmonious labeling. We suggest that there is an open problem for further research as follows. 
Open Problem. Find if there exists odd harmonious labeling on pleated of the Dutch windmill graph $C_{n}^{(k)}(r)$ with $n \equiv 0(\bmod 4), k \geq 1$ and $r \geq 1$.

\section{REFERENCES}

[1] J. A. Gallian, "Dynamic Survey of Graph Labeling," The Electronics Journal of Combinatorics, vol. 19, pp. 1-408, 2016.

[2] R. L. Graham and N. J. A. Sloane, "On Additive Bases and Harmonious Graphs," SIAM J. Algebra. Disc. Math, vol. 1, no. 4, pp. 382-404, 1980.

[3] Z. H. Liang and Z. L. Bai, "On the Odd Harmonious Graphs with Applications," J Appl Math Comput, vol. 29, pp. 105-116, 2009.

[4] F. Alyani, F. Firmansah, W. Giyarti and K. A. Sugeng, "The Odd Harmonious Labeling of KCn-Snake Graphs for Spesific Values of $\mathrm{n}$, that is, for $\mathrm{n}=4$ and $\mathrm{n}=8$," in IndoMS International Conference on Mathematics and Its Applications IndoMS and UGM, Yogyakarta, 2013.

[5] F. Firmansah, "Pelabelan Harmonis Ganjil pada Gabungan Graf Ular dan Graf Ular Berlipat," in Konferensi Nasional Matematika dan Pembelajarannya (KNPMP 1) UMS, Solo, 2016.

[6] F. Firmansah and K. A. Sugeng, "Pelabelan Harmonis Ganjil pada Graf Kincir Angin Belanda dan Gabungan Graf Kincir Angin Belanda," Magistra, vol. XXVII, no. 94, pp. 56-92, 2015.

[7] F. Firmansah and M. W. Syaifuddin, "Pelabelan Harmonis Ganjil pada Graf Kincir Angin Double Quadrilateral," in Seminar Nasioanal Matematika dan Pendidikan Matematika UNY, Yogyakarta, 2016.

[8] M. E. Abdel-Aal, "Odd Harmonius Labeling of Cyclic Snakes," International Journal on Applications of Graph Theory in Wireless Adhoc Networks and Sensor Networks, vol. 5, no. 3, pp. 1-13, 2013.

[9] M. E. Abdel-Aal, "New Families of Odd Harmonious Graphs," International Journal of Soft Computing, Mathematics, and Control, vol. 3, no. 1, pp. 1-13, 2014.

[10] A. G. Saputri, K. A. Sugeng and D. Froncek, "The Odd Harmonious Labeling of Dumbell and Generalized Prism Graphs," AKCE Int. J. Graphs Comb, vol. 10, no. 2, pp. 221-228, 2013.

[11] P. Jeyanthi and S. Philo, "Odd Harmonious Labeling of Some Cycle Related Graphs," Proyecciones Journal of Mathmatics, vol. 35, no. 1, pp. 85-98, 2016.

[12] P. Jeyanthi, S. Philo and K. A. Sugeng, "Odd Harmonious Labeling of Some New Families of Graphs," SUT Journal of Mathematics, vol. 51, no. 2, pp. 53-65, 2015.

[13] S. K. Vaidya and N. H. Shah, "Odd Harmonious Labeling of Some Graphs," International J. Math. Combin, vol. 3, pp. 105-112, 2012.

[14] S. K. Vaidya and N. H. Shah, "Some New Odd Harmonious Graphs," International Journal of Mathematics and Soft Computing, vol. 1, no. 1, pp. 9-16, 2011. 